\title{
Research on the Output Characteristics of Emerging Market Entities Serving the Development of New Energy
}

\author{
Shuo Yin ${ }^{1}$, Xing Chen ${ }^{1}$, Zhe Chai ${ }^{1}$, Yao Lu ${ }^{1}$, Danni Zhang ${ }^{2, *}$ \\ ${ }^{1}$ State Grid Henan Electric Power Company Economic and Technological Research Institute, Zhengzhou, China \\ ${ }^{2}$ School of Electrical Engineering, Zhengzhou University, Zhengzhou, China
}

\begin{abstract}
The power emerging market entities connected to the grid in a decentralized manner can increase the local new energy consumption rate. Under the background of accelerating the development of new energy, it is urgent to clarify the output characteristics of emerging market entities such as wind power, photovoltaics, energy storage, and electric vehicles to adapt to the development of distributed energy and the progress of power market reform. Major emerging entities contribute to mathematical analysis, promote the transaction design of emerging entities, and promote the rapid and healthy development of new energy.
\end{abstract}

Keywords: New energy; Emerging market; Electricity trading; Output characteristics

\section{Introduction}

The realization of the carbon peak, carbon neutral target and the goal of building a new power system with new energy as the main body requires the number of new energy installed capacity and the development of power market to guarantee. At the same time, as China's power market reform enters the deep water area, the specific circumstances in the transaction process after the power sale side is liberalized are very complicated, and will also face a variety of risks. The output characteristics of emerging entities such as wind power, photovoltaics, and energy storage are clarified. It is the key to the penetration of the new power system into the traditional power system.

\section{Wind power output characteristics}

Performance characteristics of wind power output curve: (1) Randomness. From the fitting curve of the annual output curve of wind power, it can be clearly observed that for the hourly output of wind power, the output at each moment shows obvious uncertainty.

(2) Intermittently. The wind speed is obviously intermittent, and it is also affected by the cutting-in speed and the cutting-out speed. Therefore, there are some points in the curve where the output of the fan is zero. The point where the output of these fans is zero may be due to the fact that the cut-in wind speed has not been reached, or the cut-out wind speed has been reached, and the fan has been cut off, so the fan output is not continuous.

(3) Seasonal characteristics. The output of wind power has certain seasonal variation characteristics. The output of wind power is the largest in winter, and the output of wind power in autumn is the smallest. The difference is relatively obvious. At the same time, the output curve of each season is quite different from the typical sunrise curve, and the typical sunrise curve cannot well reflect the characteristics of the output change in each season.

To study the characteristics of wind power output, we must first establish a mathematical model of wind speed. A large amount of literature shows that the mathematical model of wind speed (that is, the distribution of wind speed) fits well with the two-parameter Weibull distribution. The mathematical function expression of the two-parameter Weibull distribution is as follows:

$$
H(V)=\left(\frac{\beta}{\alpha}\right)\left(\frac{v}{\alpha}\right)^{\beta-1} \exp \left[-\left(\frac{v}{\alpha}\right)^{\beta}\right]
$$

Among them, $v$ represents the wind speed; $\beta$ represents the shape parameter; $\alpha$ represents the size parameter. Similarly, the probability distribution function of the twoparameter Weibull is as follows:

$$
G(v)=\int_{0}^{+\infty} H(v) d v=1-\exp \left[-\left(\frac{v}{\alpha}\right)^{\beta}\right]
$$

In a more ideal state, each group of wind speed data has a unique power corresponding to it, but the relationship between wind speed and power is affected by the characteristics of the wind turbine itself in engineering. The wind speed-power characteristic curve is shown in Figure 1(left):

\footnotetext{
* ${ }^{*}$ orresponding author: yinshuo1111@163.com
} 

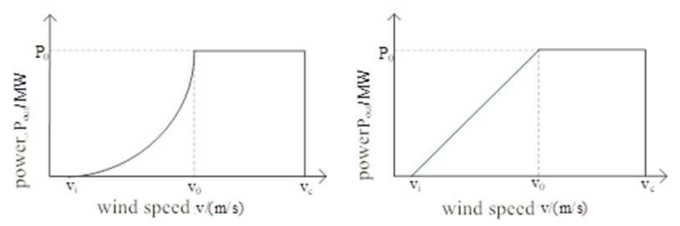

Figure 1 Wind speed-power curve

In engineering applications, the linear curve in Figure 1 (right) can be used to replace the non-linear curve, which makes it easier to obtain the required data. When using the wind speed-power linear curve, the mathematical expression of the fan output power section function is as follows:

$$
\begin{aligned}
& P_{0}=\pi R^{2} \rho v^{3} \frac{\left(1+\frac{v_{0}}{v}\right)^{2}\left(1-\frac{v_{0}}{v}\right)}{4} \\
& P_{\text {out }}= \begin{cases}0, & 0 \leq v \leq v_{i} \\
\frac{v-v_{i}}{v_{0}-v_{i}} P_{0}, & v_{i}<v \leq v_{0} \\
P_{0}, & v_{0}<v \leq v_{c} \\
0, & v>v_{c}\end{cases}
\end{aligned}
$$

Among them, $P_{0}$ is the rated output of the wind turbine; $v_{i}$ is the cut-in wind speed of the fan; $v_{0}$ is the rated wind speed; $v_{c}$ is the cut-out wind speed; $v$ is the wind speed at the height of the wind turbine hub; $R$ is the fan radius; $\rho$ is the air density where the fan is located.

\section{Photovoltaic output characteristics}

Distributed photovoltaic power generation has the characteristics of strong intermittent and strong randomness, and will be affected by environmental conditions and other factors, such as: season, geographic area, time, etc. It will also form a power load restriction with the connected neighboring photovoltaic. The output level of photovoltaic power generation is closely related to the changes in the solar radiation received by the ground. The output level of the photovoltaic power generation system increases first and then decreases with the change of solar radiation, reaching the peak output at noon, showing a "semi-envelope" shape overall. The width of the half envelope is related to the sunshine time, and the height of the half envelope is related to the season and meteorological conditions. For this reason, the output characteristics of photovoltaic power generation can be discussed from the following two aspects.

(1) The influence of seasonal attributes

The time period of photovoltaic power output is limited by the time of sunrise and sunset, so it is greatly affected by the characteristics of the season. The shape of the photovoltaic output curve is quite different. The initial power generation time of the photovoltaic power station in summer and winter differs by up to 2 hours, and the difference in the duration of the full-day output is close to 4 hours.

Generally speaking, "sunrise time plus $0.5 \mathrm{~h}$ " can be used to characterize the start time of photovoltaic power generation on the day, and "sunset time minus $0.5 \mathrm{~h}$ " can be used to characterize the end time of power generation. It can generally be considered that in a certain season, the time of sunrise and sunset of a certain place are basically unchanged. Therefore, for the photovoltaic output curve, the corresponding seasonal attributes are very important. If the season attributes are known, the start time and end time of photovoltaic power generation can be roughly determined according to the time of sunrise and sunset, and the duration of photovoltaic power generation can be further calculated.

(2) The influence of meteorological conditions Because photovoltaic power generation is directly affected by solar radiation, different weather conditions will also affect the output level of the photovoltaic power generation system. For example, under rainy or cloudy weather conditions, the output of the photovoltaic power generation system may drop sharply. This brings greater uncertainty to the output of photovoltaic power generation systems. Weather conditions have a great influence on the output of photovoltaic systems and are an important constraint on the output of photovoltaic systems.

There are many methods to study the output characteristics of photovoltaic power generation in engineering, but the main method is to use the multivariable nuclear density estimation method to establish a mathematical model of photovoltaic power generation output for characteristic analysis and the project uses this method to believe that this method conforms to the Bata distribution.

The output of photovoltaic power generation is mainly affected by the light intensity. The standardized light intensity is as follows:

$$
H_{t}=\frac{I_{t}}{I_{\max }}
$$

Among them, $I_{t}$ is the actual light intensity; $I_{\max }$ is the maximum light intensity; according to the principle of Bata distribution, its probability density function is

$$
F_{H_{t}}\left(H_{t}, \gamma, \theta\right)=\frac{\Gamma(\gamma+\theta)}{\Gamma(\gamma) \Gamma(\theta)} H_{t}^{\gamma-1}\left(1-H_{t}\right)^{\theta-1}
$$

Among them, $\gamma, \theta$ are the shape parameters of Bata,

$$
\Gamma(.) \text { is the gamma function, } \Gamma(\gamma)=\int^{\infty} x^{\gamma-1} e^{-x} d x \text {. }
$$

Photovoltaic power plant receives solar radiation and converts it into usable electric energy, then the light energy-power conversion can be calculated by the following formula:

$$
P_{\text {out }}=\lambda S I\left[1-0.005\left(t_{0}+25\right)\right]
$$

Among them: $\lambda$ is the conversion efficiency of light energy and electric energy; $S$ is the area of the 
photovoltaic panel; $I$ is the solar irradiance; $t_{0}$ is the ambient temperature where the photovoltaic device is installed.

\section{Energy storage output characteristics}

This section takes the static energy storage device lithium battery as an example to analyze. In the discharge process of the lithium battery, it uses constant current discharge. The change of the working voltage in this process can be divided into 3 stages. In the initial stage of discharge, the battery working voltage drops rapidly, and then enters the linear drop zone. When the discharge is close to the end, the battery working voltage begins to drop again. When the voltage is lower than a certain value, the discharge stops. Combining with the charging and discharging characteristics of lithium batteries, it can be seen that during the charging and discharging stage, the charging and discharging power of lithium batteries is constantly changing. Combining with the charging and discharging characteristics of lithium batteries, it can be seen that during the charging and discharging stage, the charging and discharging power of lithium batteries is constantly changing, in order to play the role of peak shaving and valley filling after being connected to the large power grid, it is necessary to rationally arrange the electrochemical energy storage capacity in combination with the peak load of the power grid.

In order to explore the distribution characteristics of the battery during charging and discharging more in-depth, this study uses a variety of distribution functions to fit the data of the battery during charging and discharging, and determine the best fitting distribution. This study uses the distribution in the @ risk software The fitting function is used to specifically analyze the quantitative characteristics of the battery during charging and discharging, and the probability distribution of the battery during charging and discharging under certain conditions can be obtained. At the same time, the Anderson-Darling (A-D) test was performed on the fitting results. The test results showed that among the many distribution functions, the A-D test value of the Logistic distribution is the smallest, that is, the logistic distribution has the highest degree of fit, so it can be judged that the battery charge and discharge roughly obey Logistic distribution, the specific probability density function expression is shown in the following formula:

$$
F(x)=\frac{e^{\frac{x-\alpha}{\beta}}}{\beta\left(1+e^{\frac{x-\alpha}{\beta}}\right)^{2}}
$$

Where, $F(x)$ represents the probability that the sample data is near a certain value point, $\alpha$ represents the average value of the data (location parameter), $\beta$ represents the variance (scale parameter). In the process of sample fitting, $\alpha, \beta$ need to be estimated based on different sample data. According to the collected data, the
Monte Carlo simulation (5000 simulations) is performed, and the logistic probability density function of the charge and discharge output of the battery is fitted. The mean parameter is 0.21 and the variance parameter is 0.89 . There may be differences in the probability distribution functions or related parameters obtained by fitting different types of chemical energy storage.

\section{Electric vehicle output characteristics}

Conventional electric vehicle charging and discharging control uses each electric vehicle and its battery status as research variables, and the simulation process is relatively complicated, while the electric vehicle switching mode can focus on the modeling of the switching station itself, which is equivalent to an energy storage device. Modeling the energy storage state in adjacent periods. On the one hand, the power exchange station interacts with the power grid through the charging pile, and on the other hand supplies electric energy to the electric vehicle in the form of battery pack replacement. The total remaining power in the station at time $\mathrm{t}$ can be expressed as:

$$
Q_{t}=Q_{t-1}+\left(P_{t}^{c} \eta_{c}-\frac{P_{t}^{d}}{\eta_{d}}\right) \Delta t-D_{t-1}\left(w-L_{t-1}\right)
$$

Among them: $Q_{t}$ represents the remaining electric energy of the switching station at time $\mathrm{t} ; P_{t}^{d}$ represents the power injected into the switching station from the grid at time $t$; $\eta_{c}$ represents the power fed into the grid by the switching station at time $\mathrm{t} ; \eta_{d}$ represents the charging efficiency of the switching station receiving power from the grid; $D_{t}$ represents the number of electric vehicle battery packs that need to be replaced in the t time period; $w$ represents the rated capacity of the battery pack; $L_{t}$ Represents the average remaining power of the battery packs replaced in the $\mathrm{t}$ time period.

Considering the charging and discharging status of the entire swap station, assuming that the swap station will reserve a charging position for the battery pack replaced by an electric vehicle at time $t$, according to the power swap demand, the charge and discharge power constraints of the swap station at time $\mathrm{t}$ can be obtained as:

$$
\begin{aligned}
& 0 \leq P_{t}^{c} \leq c_{t}\left(M-D_{t}\right) P_{b}^{c} \\
& 0 \leq P_{t}^{d} \leq d_{t}\left(M-D_{t}\right) P_{b}^{d}
\end{aligned}
$$

Among them: $P_{b}^{c}$ represents the charging power of the battery pack; $P_{b}^{d}$ represents the discharge power of the battery pack; $M$ represents the total number of charging positions in the switching station; $c_{t}, d_{t}$ represent the charging and discharging control variables $(0 / 1)$ at time $t$, and the two variables are mutually exclusive.

Assuming that both the battery swap station and the electric vehicle use battery packs with a uniform standard capacity, in order to ensure the charging demand in a certain period of time in the future, set the lower limit of 
the remaining power at time $t$ in the station as, and the power storage limit and reserve demand of the swap station can be expressed as:

$$
\left(1+\eta_{e v}\right) Q_{\min , t} \leq Q_{t} \leq w M_{B}
$$

Among them: $\eta_{e v}$ stands for the power reserve rate in the swap station; $M_{B}$ stands for the number of backup batteries in the swap station.

\section{Conclusion}

The power emerging market entities connected to the grid in a decentralized manner can increase the local new energy consumption rate. Distributed energy not only improves the utilization level of new energy, but also brings huge economic and environmental benefits, and helps to achieve sustainable economic and social development. With the expansion of power emerging market entities and the implementation of market-oriented trading trials, it is necessary to accurately evaluate and analyse the economic and environmental benefits of power emerging market entities. Therefore, accurate evaluation models need to be established to guide grid companies and government departments to better develop and utilize the main technology of the power emerging market. From an overall point of view, clarifying the output characteristics of the power emerging market entities will help to connect to the large power grid and help save investment, reduce energy losses, and give full play to the respective advantages of distributed power sources and power grids. From the perspective of the main projects in the power emerging market, it will also help investors to build costs, make reasonable investments, and provide accurate quotations.

\section{Acknowledgments}

This work was financially supported by the State Grid Henan Electric Power Company Science and Technology Project (5217L021000F).

\section{References}

1. Yang Bai, Ang Li, Qing Xia, Electricity marketing model and new electricity price system under the new situation, J. Power System Protection and Control.2016,44(05):10-16.

2. Yang Bai, Le Xie, Qing Xia, Qixin Chen, Haiwang Zhong, China's system design and suggestions for promoting the marketization of the power sales side, J. Automation of Electric Power Systems, 2015,39(14):1-7.

3. Chengshan Wang, Zhen Wu, Peng Li, Application prospects and challenges of distributed electric energy storage technology, J. Automation of Electric Power Systems, 2014,38(16):1-8+73.

4. Shiming Tian, Beibei Wang, Jing Zhang, Key technologies of demand response under smart grid conditions, J. Proceedings of the Chinese Society for Electrical Engineering, 2014,34(22):3576-3589.

5. Li Ma, Menghua Fan, Lei Guo, Song Xue, Kun Li, The latest development trend of foreign power market and its enlightenment, J. Automation of Electric Power Systems, 2014,38(13):1-9.

6. Jiajia Yang, Junhua Zhao, Fushuan Wen, Yusheng Xue, Liang Li, Haohua Lv, Bidding strategy of virtual power plant including electric vehicles and wind turbines, J. Automation of Electric Power Systems, 2014,38(13):92-102. 